\title{
Editorial
}

William J: Greeley, Frank H. Kern, Jon Meliones, Ross M. Ungerleider

\section{Monitoring the brain during cardiac surgery in children}

blood flow velocity (CBFV), cerebral perfusion pressure (CPP) and the relationship of CBFV and CPP before and after deep hypothermic CPB (DHCPB) with fullflow $\left(2.4 \mathrm{~L} \cdot \mathrm{m}^{-2} \cdot \mathrm{min}^{-1}\right)$, continuous low-flow DHCPB (CLF-CPB), where CLF-CPB is defined by the authors as pump flow rates less than or equal to 1.2 $\mathrm{L} \cdot \mathrm{m}^{-2} \cdot \min ^{-1}$ ), and DHCA. TCD technology uses the Doppler principle to detect shifts in the frequency of reflected signals from blood in the middle cerebral artery to calculate blood flow velocity. Since the diameter of this large cerebral artery is relatively constant, flow velocity should approximate cerebral blood flow. In concert with AFP monitoring, TCD is one of a number of methods currently being used to monitor cerebral blood flow during paediatric cardiac surgery.

The principle advantages of TCD include: (1) it is noninvasive, (2) it does not require radiation exposure and (3) it is a continuous monitor. An additional advantage of this technique is the capability of assessing rapid alterations in blood flow velocity due to temperature or perfusion changes, as commonly occur during cardiac surgery. The limitations of transcranial Doppler monitoring include: (1) reproductibility, especially at low flows, where minute movement of the patient's head can dramatically alter signal intensity and alter baseline measurements, (2) the lack of validating studies of TCD during hypothermic $\mathrm{CPB}$, where temperature, reduced flow rates and the laminar flow characteristics of nonpulsatile perfusion, may limit the accuracy of cerebral blood flow velocity measurements. While CBFV measurements by TCD have reasonable correlation with more standard measures of CBF during normothermia, there have been few studies examining its validity during hypothermic cardiopulmonary bypass. ${ }^{3}$ Burrows and Bissonnette succinctly discuss some of these limitations of TCD. Nonetheless, published reports using this technique, ${ }^{4}$ including the present study, bear remarkable similarity to the studies of direct CBF measurements using other methods during cardiac surgery by other groups. ${ }^{5}$

The primary goal of brain monitoring is to improve our understanding of the mechanisms of injury during 
cardiac surgery so that effective brain protection strategies can be developed. Because many of the determinants of normal brain perfusion become externally controlled by the cardiac team during CPB, e.g., flow rate (cardiac output), perfusion pressure, temperature, haematocrit and $\mathrm{PaCO}_{2}$, a knowledge of the effect of these factors on the brain in neonates, infants and children, is essential. Furthermore, the examination of the brain under unusual biological circumstances, such as after DHCA or during CLF-CPB at deep hypothermia $\left(16-18^{\circ} \mathrm{C}\right)$ permits an unique opportunity to describe cerebrovascular physiology and pathophysiology. Recent studies examining the brain during cardiac surgery, such as that reported in this issue by Burrows and Bissonnette, have enabled several investigative groups to provide important information regarding questions of normal and abnormal brain perfusion during cardiac surgery in children.

We now know that during cardiopulmonary bypass in children, temperature is linearly related to cerebral blood flow (CBF) and cerebral metabolism $\left(\mathrm{CMRO}_{2}\right)$ is exponentially related. ${ }^{5-6}$ Further, anaesthetic death, $\mathrm{PaCO}_{2}$, and perfusion pressure are major factors that regulate $\mathrm{CBF}$ and $\mathrm{CMRO}_{2}$ during $\mathrm{CPB} .{ }^{7}$ Importantly, it has been shown that under deep hypothermic conditions $\left(16-18^{\circ} \mathrm{C}\right)$, such as that studied by Burrows et al. cerebral blood flow becomes dysautoregulated and the blood flow/metabolism ratio becomes uncoupled. ${ }^{6}$ Because $\mathrm{CBF}$ linearly decreases with temperature and $\mathrm{CMRO}_{2}$ decreases exponentially, blood flow is luxuriant under conditions of deep hypothermia. It now also appears, both in experimental models and in humans, that after DHCA there is an impairment in $\mathrm{CBF}$ and $\mathrm{CMRO}_{2}{ }^{4,5,8}$ In human infants after DHCA, oxygen availability in brain tissue is sufficient; however, mitochondrial utilization of oxygen stores is disordered. ${ }^{9}$

In their investigation, Burrows and Bissonnette examined the effect of low-flow cardiopulmonary bypass and circulatory arrest on cerebral pressure-flow velocity relationship: does DHCA alter the cerebral perfusion pressure required to re-establish cerebral blood flow? The authors demonstrated that after DHCA, the required cerebral perfusion pressure was higher. They postulate the interesting concept of an altered critical opening pressure after DHCA. Their finding may explain the hypoperfusion state demonstrated after DHCA and adds further to our knowledge regarding the effects of DHCA. Whether the abnormalities in CBFV as measured by TCD are linked to neuropsychological dysfunction remains to be determined.

The surgical alternative to DHCA is continuous, lowflow CPB (CLF-CPB) at deep hypothermic temperatures. This technique has been advocated as a method to mitigate the brain abnormalities and neuropsychological dys- function observed after DHCA. It has been hypothesized that the provision of continuous oxygen and substrate delivery during CLF-CPB would be beneficial because of the inherent luxury perfusion during deep hypothermia. Recent preliminary experimental evidence suggests the superiority of CLF-CPB over DHCA. ${ }^{8}$ The authors also investigated the effects of CLF-CPB by TCD. In their study they noted that during DHCPB, cerebral blood flow became dysautoregulated. This confirms previous studies examining the same issue. ${ }^{5}$ The discrepancies regarding blood flow after CLF-CPB by the authors and other reports may be related to the different methods of measurement (TCD vs $\mathrm{Xe}^{133} \mathrm{CBF}$ ) and site of investigation (TCD assesses the middle cerebral artery and $\mathrm{Xe}^{133}$ assesses the microcirculation of the brain).

Of interest, the authors found a subset of patients in the CLF-CPB group whose TCD recovery was similar to the DHCA patients. They postulate that in these patients the critical opening pressure exceeded perfusion pressure during CLF-CPB, causing vessel collapse and a cerebrovascular state identical to DHCA. Although an interesting explanation, it seems unlikely that a reduction in pump flow rates to $50 \mathrm{ml} \cdot \mathrm{kg}^{-1} \cdot \mathrm{min}^{-1}$ is severe enough to cause collapse and the absence of flow in the middle cerebral artery. The reduced perfusion seen after low-flow bypass in five of the 17 CLF-CPB patients most likely is a marker for ischaemic injury incurred during the lowflow period. Although $50 \mathrm{ml} \cdot \mathrm{kg}^{-1} \cdot \mathrm{min}^{-1}$ should be more than adequate flow for temperatures of $20^{\circ} \mathrm{C}$, the most likely cause for ischaemic injury is inadequate flow to meet cerebral metabolic requirements. Several recent studies support the concept that short cooling times or variations in cooling techniques prior to flow reduction may not allow for homogenous cerebral cooling and may be a major source for cerebral ischaemic injury. $6,10,11$

The paper seems to have reasonable controls, but only five patients in a subgroup that is prone to extreme biological variability. Disease entities alone can lead to different patterns of cerebral perfusion both before and after $\mathrm{CPB}$. Both the control group and other subgroups may be selected by nature of disease entities, rendering study data inconclusive. This is a problem in clinical studies and needs to be appreciated by the authors when interpreting their data. What are the differences between patient groups and can these differences explain some of the TCD findings?

As with all of the studies assessing cerebral blood flow and cerebral blood flow velocity, the scientific emphasis has been on answering questions regarding cerebral vascular reactivity and cerebral autoregulation. These were important and logical first questions to answer, but now more complex problems need to be addressed. It is, after all, not just cerebral blood flow or cerebral blood flow 
velocity, but the appropriateness of oxygen supply to demand that is crucial. Although measures of CBF are important, cerebral metabolism remains the single most important aspect of monitored cerebral physiology during CPB. This is reflected in the Burrows and Bissonnette's investigation examining the effect of low-flow CPB and circulatory arrest on cerebral pressure-flow velocity relationship. The authors were unable to distinguish inadequate cerebral perfusion in a small subset of patients undergoing CLF-CPB until abnormalities in reperfusion were detected in the post-bypass period. This means that measurements of CBF or CBFV in isolation may not provide adequate insight into cerebral well-being. Furthermore, by measuring flow in large cerebral vessels, the authors may be missing significant flow that is nonpulsatile in the microcirculation, but which is still being delivered to important parts of the cerebral vasculature. It is difficult to understand the meaning of these data without matched metabolic information.

Abnormalities in cerebral perfusion, reflected by a flattening in the pressure-CBFV curve after an ischaemic period (DHCA and 5/17CLF-CPB patients) are an important observation and adds further to our knowledge regarding DHCA. It suggests that patients have marginal cerebral perfusion in the post-DHCA period and are vulnerable to a "second neurological injury" at normothermic temperatures. We know that the post-CPB period is a period of reduced oxygen delivery. Reduced cardiac output, reduced haematocrits and pulmonary dysfunction are a common problem after weaning from $\mathrm{CPB}$. If cerebral perfusion is impaired after DHCA, marginal cerebral perfusion may become inadequate in the face of significant cardiac or pulmonary dysfunction. In either case, increasing cerebral oxygen delivery possibly through improved CPP or cardiac output may be beneficial although this remains speculative.

Thus, the larger question is what are the effects of CPB techniques on cerebral metabolism? The fact that transcranial Doppler merely assesses perfusion limits its usefulness as a tool to further assess low-flow cardiopulmonary bypass or circulatory arrest. While changes in blood flow patterns are real and often dramatic, metabolism is more important and blood flow alterations have not been linked with poor outcome.

Importantly, the immediate cerebrovascular findings, such as those reported by Burrows and Bissonnette, must be coupled with sensitive measures of neuropsychological outcome before we can have the necessary information regarding the many questions concerning cerebral blood flow velocity and $\mathrm{CPB}$. Only then can we answer the vital question: to what degree does impairment of cerebral blood flow velocity measured by TCD contributed to postoperative neuropsychological dysfunction?
Although neuropsychological dysfunction is the best and only real determinant of outcome, the difficulty in obtaining such data in children, the expense required, and the long waiting period for results (3-7 yr) renders this outcome measure restrictive and possibly confounding in linking cause with effect. More immediate outcome measures are necessary to determine useful cause-effect relationship. This will require careful studies linking immediate outcome measures, such as those reported by the authors using transcranial Doppler, with long-term neuropathological or neuropsychological changes. Identification of useful immediate measures of cerebral outcome can then be used to assess potentially useful strategies of brain protection, whether they be perfusion or pharmacological in origin.

Finally, another important feature of TCD is the ability to detect cerebral emboli in the middle cerebral artery, which is beyond the scope of the study by Burrows $e t$ al. Quantification of this important mechanism of cerebral injury during cardiac surgery would be instructive. Future investigations using TCD should address this mechanism of injury as well. Likewise, the evaluation of the internal carotid artery by TCD should be evaluated as a more comprehensive approach to the problem of cerebral emboli and cardiac surgery.

The study by Burrows et al. should not cause surgical teams to avoid DHCA or continuous low-flow CPBmetabolic data, including that from our group, are favourable in support of the judicious application of these techniques; plus it works - infants do well with it. The challenge is to improve cerebral protection. The results of Burrows et al. may add important information that can help us find ways to make our CPB strategies better. It is anticipated that with greater understanding of the mechanisms of CBF and cerebral metabolism regulation, progress will surely come towards better cerebral protection during cardiac surgery in infants and children.

\section{Le monitorage cérébral pendant la chirurgie cardiaque chez l'enfant}

C'est grâce à l'amélioration des techniques chirurgicales et à la protection myocardique que nous obtenons maintenant d'excellents résultats dans la correction des malformations cardiaques congénitales complexes. Le taux de mortalité pour ce type de chirurgie n'a cessé de décrồ- 
tre pendant la dernière décennie. Cependant à mesure que s'améliore le pronostic immédiat, le dysfonctionnement neuropsychologique de l'opéré pour malformation congénitale se présente comme une complication de plus en plus fréquente et de plus en plus manifeste. Comme les nouveaux-nés sont souvent exposé à des températures exceptionnellement basses $\left(15-18^{\circ} \mathrm{C}\right)$ et à des déviations extrèmes de la perfusion normale (arrêt circulatoire complet) pendant la chirurgie correctrice, la nature du pronostic neurophysiologique à long terme devient un sujet d'inquiétude majeur. Il est donc logique que l'on commence à examiner la qualité de vie de ceux qui survivent à ces interventions.

Des études récentes portent à croire que $25 \%$ des enfants qui ont subi une CEC sous hypothermie avec arrêt circulatoire complet souffrent de déficiences neurologiques transitoires et permanentes. ${ }^{1}$ Lors d'interventions palliatives complexes comme la correction de l'hypoplastie ventriculaire gauche, le déficit neurologique frappe jusqu'à $45 \%$ des patients. ${ }^{2}$ On ne connait pas avec certitude les causes des lésions neurologiques, mais elle semblent provenir de plusieurs mécanismes: l'arrêt circulatoire en hypothermie profonde, l'embolie gazeuse cérébrale, la façon de conduire la CEC et plus spécialement, le refroidissement et le réchauffement. Comme cette conduite chez l'enfant diffère considérablement de celle de l'adulte, il est nécessaire de mieux comprendre les effets cérébraux de la CEC et des techniques qu'elle comprend pendant la chirurgie cardiaque infantile. Il n'est pas surprenant de réaliser que l'étude des mécanismes lésionnels et des plans d'attaque pour les contrer, occupent une grande place de la recherche pédiatrique courante sur la CEC.

Dans ce numéro, Burrows et Bissonnette exposent leur expérience sur l'utilisation du Döppler transcrânien (DTC) et les mesures de la pression de la fontanelle antérieure (PFA) dans le but évaluer la vélocité du débit sanguin cérébral (VDSC), la pression de perfusion cérébrale (PPC) et le rapport entre VDSC et PPC avant et après CEC sous hypothermie profonde à plein débit $\left(2,4 \mathrm{~L} \cdot \mathrm{m}^{-2} \cdot \mathrm{min}^{-1}\right)$, sous hypothermie profonde à bas débit continu $\left(\geq 1,2 \mathrm{~L} \cdot \mathrm{m}^{-2} \cdot \mathrm{min}^{-1}\right)$, et l'arrêt circulatoire en hypothermie profonde. L'ultrasonograhie Döppler utilise l'effet Döppler pour détecter les modifications de fréquences des signaux réfléchis par le sang de l'artère cérébrale moyenne permettant ainsi de calculer la vélocité du courant sanguin. Comme le calibre de cette artère est relativement constant, la vélocité du débit devrait s'approcher de la mesure du débit sanguin cérébral. Le DTC, de concert avec le monitorage de la PFA, est une des méthodes couramment utilisées pour monitorer le débit sanguin cérébral pendant la chirurgie cardiaque pédiatrique.
Les principaux avantages du DTC se définissent comme suit: 1) c'est un moniteur non invasif, 2) il n'expose pas aux radiations, 3) et il est continu. De plus, il est capable d'évaluer les modifications rapides de la vélocité du courant sanguin causées par les changements de perfusion et de températures inévitables en chirurgie cardiaque. Le DTC a aussi ses limitations: la reproductibilité, surtout à bas débit, lorsque des mouvements minimes de la tête peuvent changer de façon spectaculaire l'intensité du signal et la ligne de base, 2) l'absence de validation du DTC pendant la CEC hypothermique: au cours de celle-ci, la température, les bas débits et les caractéristiques du courant laminaire de la perfusion non pulsatile gênent la précision des mesures de VDSC. Alors que les mesures de VDSC par Döppler transcrânien présentent une corrélation acceptable avec les mesures réalisées avec les méthodes usuelles pendant la normothermie, peu d'études se sont penchées sur sa validité pendant l'hypothermie. ${ }^{3}$ Burrows et Bissonnette discutent brièvement des limitations du DTC. Néanmoins, on trouve une ressemblance remarquable entre les études ${ }^{4}$ qui utilisent cette technique, incluant la présente, et les mesures directes du DSC par d'autres méthodes pendant la chirurgie cardiaque. ${ }^{5}$

On utilise le monitorage cérébral pour comprendre le mécanisme des lésions nerveuses pendant la chirurgie cardiaque et pour développer des méthodes de protection cérébrale. Comme la plupart des facteurs qui déterminent le débit sanguin cérébral tombent sous le contrôle de l'équipe de la CEC pendant la chirurgie, comme le débit, la pression de perfusion, la température l'hématocrite et la $\mathrm{PaCO}_{2}$, il faut connaître l'influence de ces paramètres sur le cerveau du nouveau-né, du nourrisson et de l'enfant. De plus, sous des conditions biologiques aussi exceptionnelles que l'arrêt circulatoire en hypothermie profonde ou la CEC à bas débit hypothermique $\left(16-18^{\circ} \mathrm{C}\right)$, l'examen du cerveau offre l'opportunité unique d'observer la physiologie et la physiopathologie cérébrovasculaires. Des études récentes comme celle-ci, permettent aux investigateurs d'obtenir des renseignements importants sur la perfusion normale et anormale pendant la chirurgie cardiaque chez l'enfant.

Nous savons maintenant que pendant la CEC chez l'enfant, la relation entre la température et le DSC est linéaire, alors que la relation avec le métabolisme cérébral est exponentielle. ${ }^{5,6}$ Nous savons aussi que la "mort anesthésique ", la $\mathrm{PaCO}_{2}$ et les pressions de perfusion sont les facteurs majeurs du contrôle du DSC et du métabolisme cérébral pendant la $\mathrm{CEC}^{7}$ On a aussi montré que sous des conditions d'hypothermie profonde $\left(16-18^{\circ} \mathrm{C}\right)$, comme celles qu'ont étudiées Burrows et Bissonnette, le DSC perd son auto-régulation, ce qui conduit au découplage du mécanisme débit $/$ métabolisme. $^{6}$ 
Comme le DSC décroît de façon linéaire avec la température et que le métabolisme cérébral diminue de façon exponentielle, le débit sanguin sous des conditions d'hypothermie devient surabondant. Il semble aussi qu'après l'arrêt cardiaque en hypothermie profonde, tant sur un modèle expérimental que chez l'humain, le DSC et le métabolisme cérébral se détériorent. ${ }^{4-6}$ Chez le nourrisson après l'arrêt circulatoire en hypothermie profonde, il reste suffisamment d'oxygène cérébral disponible; c'est son utilisation par les mitochondries qui devient anarchique. $^{9}$

Dans leur étude, Burrows et Bissonnette examinent les effets de la CEC à bas débit et de l'arrêt circulatoire sur la relation entre pression cérébrale et vélocité du débit: est-ce que l'arrêt; circulatoire complet en hypothermie profonde modifié la PPC nécessaire au rétablissement du DSC? Les auteurs montrent qu'après l'arrêt circulatoire hypothermique, la pression de perfusion cérébrale requise est plus élevée. Ils émettent une hypothèse intéressante selon laquelle la pression critique d'ouverture deviendrait plus élevée après l'arrêt. Cette observation pourrait expliquer l'hypoperfusion trouvée après l'arrêt circulatoire hypothermique. Le lien entre la VDSC telle que la mesure la PFA et l'altération neuropsychologique reste à démontrer.

La CEC à bas débit avec hypothermie profonde présente une alternative à l'arrêt hypothermique. Cette technique a déjà été préconisée pour atténuer les altérations cérébrales et neuropsychologiques observées après l'arrêt circulatoire hypothermique. On a émis l'opinion que l'apport continu d'oxygène et de substrat pendant la CEC hypothermique à bas débit pourrait être utile en raison de la surabondance du débit cérébral pendant l'hypothermie profonde. Des données expérimentales récentes suggèrent la supériorité de la $\mathrm{CEC}$ hypothermique à bas débit sur l'arrêt circulatoire hypothermique. ${ }^{8}$ Ces auteurs ont aussi utilisé le DTC pour cette étude. Ils ont noté que durant la CEC hypothermique l'autorégulation du DSC cesse, ce qui confirme les résultats d'études antérieures. ${ }^{5}$ Les différences trouvées entre le débit sanguin après $\mathrm{CEC}$ à bas débit par les auteurs et d'autres études, peuvent être en rapport avec les méthodes de mesures utilisées (DTC vs DSC X ${ }^{\mathrm{e}} 133$ ) et le site étudié (le DTC mesure au niveau de l'artère cérébrale moyenne et le DSC $\mathrm{X}^{\mathrm{e}} 133$ évalue la microcirculation cérébrale).

Il est intéressant de constater que les auteurs trouvent un sous-ensemble parmi les patients opérés sous CEC à bas débit dont la récupération mesurée au DTC est indentique à celle des patients en arrêt circulatoire hypothermique. Ils proposent comme postulat que chez ces patients, la pression d'ouverture critique excède la pression de perfusion pendant la $\mathrm{CEC}$ à bas débit, produisant ainsi un collapsus vasculaire et un status cérébro- vasculaire identique à celui de l'arrêt circulatoire hypothermique. Cette explication ne manque pas d'intérêt mais il semble peu plausible qu'une réduction du débit de pompe à $50 \mathrm{ml} \cdot \mathrm{kg}^{-1} \cdot \mathrm{min}^{-1}$ soit suffisante pour créer un collapsus et arrêter l'écoulement sanguin de l'artère cérébrale moyenne. La baisse de la perfusion notée après CEC à bas débit chez cinq des 17 patients semble plutôt le résultat de l'ischémie subie pendant la période de bas débit. Bien qu'un débit de $50 \mathrm{ml} \cdot \mathrm{kg}^{-1} \cdot \mathrm{min}^{-1}$ soit plus que suffisant à $20^{\circ} \mathrm{C}$, la cause la plus plausible de l'ischémie est l'insuffisance du débit à satisfaire aux besoins métaboliques du cerveau. Plusieurs études récentes considèrent que les courtes durées de refroidissement et la variabilité des techniques pour le refroidissement ne permettent pas de refroidir de façon homogène le cerveau et pourraient être ainsi les causes majeures de la lésion cérébrale ischémique. ${ }^{6,10,11}$

Les contrôles de la présente étude sont judicieux, mais seulement cinq patients font partie du sous-ensemble sujet à une labilité biologique extrême. La nature même de la maladie peut produire des patterns différents de perfusion cérébrale avant et après CEC. Le groupe contrôle et les sous-groupes pourraient être repartis selon leur pathologie, ce qui ne permettrait pas de tirer de conclusions. Ceci demeure le problème de toutes les études cliniques et il prend une grande place quand vient le temps pour les auteurs dinterpréter leurs données. Quelle est la différence entre les groupes de patients? Ces différences expliquent-elles certains des résultats obtenus par le DTC?

Comme dans toutes les études qui évaluent le débit sanguin cérébral et sa vélocité, on s'attaque surtout aux questions que posent la réactivité vasculaire et l'autorégulation cérébrale. Logiquement, nous devons d'abord répondre à ces questions, mais il faut aussi penser à des problèmes encore plus complexes. Même si la question du débit sanguin cérébral et de sa vélocité est importante, il n'en demeure pas moins que c'est l'harmonisation de l'apport en l'oxygène avec la demande qui est la question cruciale. Bien que les mesures de DSC soient importantes, le métabolisme cérébral demeure le facteur unique le plus important concerné par le monitorage de la physiologie cérébrale pendant la CEC. Ceci se réflète dans l'étude de Burrows et Bissonnette laquelle explore linfluence de la CEC à bas débit et de l'arrêt circulatoire sur la relation pression-vélocité du débit cérébral. Ces auteurs ne peuvent détecter linsuffisance de la perfusion cérébrale dans un petit sous-ensemble de patients opérés sous CEC à bas débit avant que n'apparaissent des anomalies de la reperfusion après CEC. Ceci signifie que les mesures isolées du débit sanguin cérébral et de sa vélocité ne fournissent pas une évaluation adéquate de la fonction cérébrale. Il semble aussi qu'en mesurant le débit dans 
d'un gros vaisseau cérébral, les auteurs négligent une fraction considérable du débit non-pulsatile de la microcirculation qui continue d'alimenter une bonne partie des vaisseaux cérébraux. Il est donc difficile de comprendre la signification de ces données si on ne tient pas compte de l'apariement avec le métabolisme cérébral.

Les anomalies de perfusion cérébrale, manifestées par un aplatissement de la courbe pression-VDSC après une période dischémie (les patients opérés sous arrêt circulatoire hypothermique et cinq de 17 patients opérés sous CEC à bas débit) représentent une observation d'importance qui accroît notre connaissance de l'arrêt circulatoire hypothermique. Ceci porte à croire que la perfusion cérébrale est marginale à la période qui suit l'arrêt et devient plus vulnérable à une deuxième atteinte neurologique en normothermie. Nous savons qu'à la période post-CEC l'apport en oxygène est réduit. La diminution du débit cardiaque, de l'hématocrite et le dysfonctionnement pulmonaire sont des problèmes fréquents au sevrage de la $\mathrm{CEC}$. Si la fonction vasculaire cérébrale est lésée par l'arrêt hypothermique, la perfusion cérébrale qui était jusque là marginale peut devenir nettement insuffisante à la faveur d'une altération importante des fonctions cardiaque et pulmonaire. Dans ce cas, les bénéfices obtenus par l'augmentation du transport de l'oxygène par amélioration de la PPC ou du débit cardiaque sont de la pure spéculation.

Ainsi, la question primordiale demeure-t-elle linfluence des techniques de CEC sur le métabolisme cérébral. Le fait que le DTC n'évalue que la perfusion limite son utilité dans l'appréciation de la CEC à bas débit et de l'arrêt circulatoire. Alors que les changements de pattern de l'écoulement sanguin sont réels et souvent spectaculaires, le métabolisme est encore plus important.

Il est aussi important de noter que les données cérébrovasculaires de Burrows et Bissonnette doivent être apariées avec des mesures sensibles de l'évolution neuropsychologique avant que nous ne puissions aborder en profondeur les questions que posent la vélocité du débit sanguin cérébral et la CEC. Alors seulement nous pourrons répondre à la question essentielle: jusqu’à quel point l'altération de la vélocité du débit sanguin mesurée par DTC contribue-t-elle au dysfonctionnement neuropsychologique postopératoire?

Bien que le dysfonctionnement neuropsychologique demeure le meilleur et le seul vrai facteur déterminant le pronostic, la difficulté rencontrée à récolter des données valides chez l'enfant, le coût, et la longue période d'attente des résultats (3-7 ans) diminuent sa valeur lorqu'll s'agit de faire une relation de cause à effet. Nous avons besoin de méthodes plus rapides et plus pratiques pour établir cette relation. Faire le lien entre pronostic et altérations neuropathologiques et neuropsychologiques va nécessiter des études aussi minutieuses que celles qu'ont réalisées les auteurs sur l'utilisation du DTC. Des mesures cérébrales ponctuelles ayant une valeur prédictive pourront être alors utilisées pour établir des plans d'intervention pharmacologiques ou perfusionnels dans le but de protéger le cerveau.

Une autre caractéristique importante du DTC mais qui dépasse la portée de l'étude de Burrows et Bissonnette concerne la capacité de détection des embolies de l'artère cérébrale moyenne. Il ne fait pas de doute que la quantification de ce mécanisme important nous renseignerait beaucoup sur la genèse des lésions cérébrales pendant la CEC. Les futures recherches sur le DTC doivent étudier ce type de lésions. De la même façon, l'évaluation de la carotide interne par DTC devrait être examinée à la lumière du problème causé par l'embolie cérébrale et la chirurgie cardiaque.

L'étude de Burrows et Bissonnette ne devrait pas décourager l'utilisation de l'arrêt hypothermique ou de la CEC à bas débit. Les données sur le métabolisme, incluant celles notre groupe, favorisent l'application judicieuse de ces techniques. De toutes façons, elles fonctionnent et les enfant s'en portent bien. Le défi consiste à améliorer la protection du cerveau. Les résultats de Burrows et Bissonnette ajoutent des renseignements importants qui nous aideront à améliorer la CEC. Il est permis d'anticiper qu'avec une connaissance plus approfondie des mécanismes de régulation du DSC et du métabolisme, nous ferons des progrès importants dans le domaine de la protection cérébrale pendant la chirurgie cardiaque de l'enfant.

\section{References}

1 Ferry $P C$. Neurologic sequelae of open-heart surgery in children. An 'irritating question'. Am J Dis Child 1990; 144: 369-73.

2 Glauser TA, Rorke LB, Weinberg PM, Clancy RR. Acquired neuropathologic lesions associated with the hypoplastic left heart syndrome. Pediatrics 1990; 85: 991-1000.

3 van der Linden $J$, Wesslén $O$, Ekroth $R$, Tydén $H$, von $A h n$ H. Transcranial Doppler-estimated versus thermodilution-estimated cerebral blood flow during cardiac operations. J Thorac Cardiovasc Surg 1991; 102: 95-102.

4 Hillier S, Burrows F, Bissonnette B, Taylor R. Cerebral hemodynamics in neonates and infants undergoing cardiopulmonary bypass and profound hypothermic circulatory arrest: assessment by transcranial Doppler sonography. Anesth Analg 1991; 72: 723-8.

5 Greeley WJ, Ungerleider RM, Smith LR, Reves JG. The effects of deep hypothermic cardiopulmonary bypass and total circulatory arrest on cerebral blood flow in infants and children. J Thorac Cardiovasc Surg 1989; 97: 737-45. 
6 Greeley WJ, Kern FH, Ungerleider RM, et al. The effect of hypothermic cardiopulmonary bypass and total circulatory arrest on cerebral metabolism in neonates, infants, and children. J Thorac Cardiovasc Surg 1991; 101: 783-94.

7 Kern FH, Ungerleider RM, Quill TJ, et al. Cerebral blood flow response to changes in arterial carbon dioxide tension during hypothermic cardiopulmonary bypass in children. $\mathbf{J}$ Thorac Cardiovasc Surg 1991; 101: 618-22.

8 Swain JA, McDonald TJ, Griffith PK, Balabal RS, Clark $R E$, Ceckler $T$ : Low flow hypothermic cardiopulmonary bypass protects the brain. J Thorac Cardiovasc Surg 1991; 102: 76-84.

9 Greeley $W$, Bracey $V$, Ungerleider $R$, et al. Recovery of cerebral metabolism and mitochrondrial oxidation state are delayed after hypothermic circulatory arrest. Circulation 1991; 82: 412-8.

10 Bellinger D, Wernovsky G, Rappaport L, et al. Rapid cooling of infants on cardiopulmonary bypass adversely affects later cognitive function. Cicrulation 1988; 78: A358.

11 Hindman BJ, Dexter F, Cutkomp J, Smith T, Todd MM, Tinker JH. Brain blood flow and metabolism do not decrease at stable brain temperature during cardiopulmonary bypass in rabbits. Anesthesiology 1992; 77: 342-51. 